\title{
BENEDIKT J. COLLINET \\ Alles auf einen Klick \\ Digitale Bibelforschung in ihren Chancen \\ und Grenzen erschließen
}

\section{Login: die digitale Ära}

Gesellschaft und Wissenschaft befinden sich seit einigen Jahren in einem Paradigmenwechsel, der gerne als Eintritt in das „Digitale Zeitalter" beschrieben wird. Mit diesem Übergang werden verschiedene Phänomene verbunden, die im Heft „Digitalisierung ${ }^{\text {“1 }}$ der Bundeszentrale für Politische Bildung gut nachlesbar sind. Digitalisierung meint vom Wortsinn her zunächst einmal die Übersetzung eines analogen Gegenstandes, z. B. eines Fotos, eines Bildes oder eines Buches, in eine computergenerierte und gespeicherte Information. Dieser Übersetzungsvorgang war anfänglich auf Prozesse wie das Scannen oder Faxen bezogen, um räumliche Distanzen schneller überwinden zu können. Schon bald gelang es, technische Geräte und Software zu erzeugen, die zu wesentlich mehr im Stande waren und aus dem heutigen Leben nicht mehr wegzudenken sind, z. B. das World Wide Web (Internet), das Smartphone oder die Digitalkamera. Diese Gegenstände entwickeln sich ständig weiter und werden auch für Wissenschaft, Gesellschaft und Politik immer relevanter, was zu einigen Ambivalenzen führt, aufgrund derer man zurecht davon ausgehen kann, dass zukünftige Konflikte auf der Informationsebene ausgetragen werden. Als Beispiele dieser These kann man einige gegenwärtige Trends benennen. Im Vorfeld der Wahl von Donald Trump zum US-amerikanischen Präsidenten gab es eine Reihe sogenannter „Fake News“, also absichtlich gestreuter Falschinformationen, die als feindliche

1 bpb (2021). 
Angriffe auf die Nation gedeutet wurden. ${ }^{2}$ Aufgrund der vorhandenen Datenfülle im Internet ist es für viele Menschen kaum mehr möglich, die Seriosität ihrer Quellen zu prüfen, häufig sind diese sogar verschleiert. Dies führt zu breiter Verunsicherung, welche die Sehnsucht nach einer starken politischen Leitfigur verstärken mag, die in schwarz und weiß unterscheidet und simplifizierende Antworten auf hochkomplexe Fragen gibt. Für die Wissenschaft bedeutet dies, dass Transparenz und Seriosität sehr wichtige Kriterien sind, wenn sie sich im Internet bewegen will. Problematisch wird es, wenn das Gros der WissenschaftlerInnen versucht, ihre Thesen leichter verständlich zu machen, damit sie der interessierten Öffentlichkeit zugänglich sind und dadurch ihr Stil einfacher wird. Dies machen sich regelmäßig pseudowissenschaftliche und verschwörungstheoretische Gruppierungen wie die Querdenker oder QAnon zunutze, indem sie im gleichen Stil eigene Thesen vertreten, schwer nachvollziehbare Quellen als seriös ausweisen oder die Ergebnisse der Forschung verkürzt oder verdreht kopieren. ${ }^{3}$

Die arbeitsintensive Nutzung der Digitalisierung scheint also auf den ersten Blick für wissenschaftliche Forschung wenig interessant zu sein, außer es geht um Breitenwirkung. Tatsächlich ist es aber so, dass die Digitalisierung ein großer Gewinn ist, der ganz neue Forschungszweige, Methoden und wesentlich schnellere und präzisere Auswertungen von Daten ermöglicht. Wenn diese Daten dann noch gratis, gut verständlich und mehrsprachig ins Internet gestellt und damit weltweit verfügbar gemacht werden (open access), wird deutlich, dass die Digitalisierung demokratisierend wirkt, koloniale Ungleichheiten langsam aufwiegt, den Zugang zu Bildung und Bildungsmate-

2 Vgl. Clivaz (2017), 41-43, die unter anderem das Peer-review-Verfahren erläutert, eine Einrichtung innerhalb wissenschaftlicher Zeitschriften, die sicherstellen soll, dass die Beiträge auf seriösen Quellen basieren und nach gültigen methodischen Standards verfasst wurden.

3 Einige gute Ausführungen zu diesem Thema finden sich im nicht mehr ganz aktuellen, aber in den Grundzügen gültigen Buch von Schweiger (2017). 
rialien weltweit verbessert u. v. m. ${ }^{4}$ Die folgenden Seiten werden am Beispiel der Bibel zeigen, wie Digitalisierungsprozesse die Humanwissenschaften heute bereichern. Die ausgewählten Exempla werden jeweils veranschaulichen, wie sich der interpretative Zugang zur Heiligen Schrift von ihrer Entstehung bis heute verändert hat.

\section{Offenbarung im Wort oder in der Schrift?}

Zunächst befassen wir uns mit dem Text der Bibel und seiner Vermittlung. Das erste Medium des offenbarten Gotteswortes war die menschliche Erfahrung mit Gott. Diese wurde erst ins Wort gefasst und weitererzählt, bevor man im Lauf des ersten Millenniums v. Chr. mit dem Niederschreiben auf Schriftrollen begann, um die Geschichten so aufbewahren zu können. Dadurch ging vieles an Erzähldynamik verloren, zugleich gab es aber einen fixen Text, den man weitergeben konnte. Das Schreibmaterial war zunächst der wenig stabile Papyrus, eine auf Schilfgras basierende Vorläuferform des Papiers, welcher schnell verrottete und deshalb häufig abgeschrieben werden musste. Im Lauf der Zeit löste das Pergament, also Tierhaut, als Medium den Papyrus ab. Die Haut ließ sich zu Seiten falten und mittels Nadel und Faden zusammennähen, sodass Folianten und Codices entstanden, welche die wesentlich kompliziertern Schriftrollen auf Stöcken ablösten. Diese Erfindung ermöglichte es den ChristInnen des 1. Jahrhunderts bis in die Spätantike hinein, die Heilige Schrift des Alten und Neuen Bundes in einem einzigen und flacheren Werk zu sammeln und zusammenzubinden. Die so entstandenen Handschriften und Bücher umfassten nach einer gewissen Zeit und theologischen Streitigkeiten eine feste Gruppe von Büchern, den Kanon, der auch „Buch der Bücher“ oder einfacher „Bibel“ genannt wurde. Bis zur Entwicklung des Buchdrucks zu Beginn des 16. Jahrhunderts wurde die Bibel abgeschrieben, es änderten sich zwar Schreibmaterialien, Illustrationen und die Kommentare zur Schrift (s. u.), doch das Grundprinzip der Handschrift blieb gleich. Die Kombination aus

4 Vgl. Clivaz (2017), 48. 
Papier und dem mechanischen Buchdruck brachten eine enorme Innovation in der Produktion der Bibel und damit in den Möglichkeiten, sie zu verbreiten. Nach der Einführung der allgemeinen Schulpflicht im Verlauf des 18. und 19. Jahrhunderts konnten immer mehr Menschen lesen, sodass Familienbibeln und andere Typen in Gebrauch kamen. Das mechanische Druckverfahren wurde im Lauf der Zeit maschinenbetrieben, schließlich computergestützt und letztlich wurde die Tinte durch den Laserdrucker ersetzt. Die digitale Wende geht aber noch einen Schritt weiter. Die Bibel muss gar nicht mehr gedruckt werden, sondern ist digital vorhanden. Dies aber nicht nur auf zahlreichen Internetseiten, wie www.bibleserver.com, sondern auch als Hörbuch. ${ }^{5}$ Durch die Digitalität gelingt es also, die biblischen Geschichten wieder in ihre ursprünglichste Form zurückzuversetzen, die erzählte Erzählung, und diese zugleich haltbar zu machen. ${ }^{6}$

Was bedeutet dies in Bezug auf Bibelwissenschaft und Offenbarung? Die Online-Bibeln sind keine Bücher mehr, sondern Dokumente bzw. Dateien. Als Audio sind sie nicht einmal mehr Schrift - und doch sind sie unverkennbar das Wort Gottes, das uns in Sprache entgegentritt, nämlich einer Computersprache, die aus binären Zahlenreihen besteht, welche in Bild oder Ton gewandelt werden. ${ }^{7}$ Diese Emanzipation der Offenbarung vom Medium der Schrift erinnert uns ChristInnen daran, so Claire Clivaz, dass das Christentum seinem Wesen nach keine Buchreligion ist, sondern eine Religion, in deren

5 Vgl. Clivaz (2017), 54-56. Dieser multimediale und multimodale Vorgang hat deutliche Auswirkungen auf das „Konsumverhalten“ zur Bibel. Clivaz listet einige Studien auf, die zeigen, dass die Bibel als Hörbuch ein anderes Publikum anspricht und zugleich deutlich mehr Menschen erreicht, als vergleichbare schriftliche Formate. Eine Besonderheit mag dabei sein, dass vor allem evangelikale und charismatische Gruppen solche Bibeln entwickeln (YouVersion, YouBible, GloBible).

$6 \mathrm{Zu}$ den Herausforderungen, die ein solcher Wechsel des Mediums mit sich bringt und zu den Möglichkeiten einer digitalen Bibeltheologie vgl. den detailliert ausgearbeiteten Vorschlag von Hasse (2013).

7 Vgl. Clivaz (2017), 36, die hier unter anderem eine Reihe weiterführender Werke (van Peursen; Schmidt u. a.) listet. 
Zentrum das inkarnierte Wort Gottes steht, Jesus Christus, der sich in Wort und Tat, im Sprechen und nicht im Schreiben offenbart hat. ${ }^{8}$ Dies erinnert uns daran, dass eine Bibel, die im Schrank steht, nicht ihren Sinn und Zweck erfüllt, Gottes Wort in die Welt zu tragen, zu verkünden und zu leben.

\section{Biblische Archäologie mit Laserscanner und GPS}

Die Biblische Archäologie ist ein Produkt des Historismus und ebenso wie die anderen Geschichtswissenschaften im Lauf des 19. Jahrhunderts entstanden. ${ }^{9}$ Sie sucht nach biblischen Spuren an den Orten, die in der Bibel beschrieben sind, vor allem in Israel-Palästina, aber auch im Libanon, Syrien und Jordanien, in Kooperation mit der Ägyptologie und der Altorientalistik in den Herrschaftsgebieten der ehemaligen Hochkulturen, sowie an jenen Orten, die in den Paulusbriefen beschrieben sind. Inspiriert von den sensationellen Funden Heinrich Schliemanns in den 1870er-Jahren (Troja, Mykene) kam es zu einem regelrechten Goldrausch im Umfeld der damals noch so genannten biblischen Hilfswissenschaften, welcher zwar eine Reihe von Funden brachte, die wissenschaftlich aber noch nicht gut aufbereitet und kategorisiert waren. Die eigentliche und bis heute gewinnbringende Arbeit begann zur Zeit des „Großen Spiels“ an den Grenzen des 20. Jahrhunderts. Einer der bedeutendsten Namen ist William F. Albright

8 Vgl. Clivaz (2017), 38; 41. Dies bedeutet allerdings nicht, dass sich die Exegese nun einfach durch einen Algorithmus ersetzen lässt, der die digitalen Signale deuten lernt. Dies ist zwar möglich, doch auch ein Algorithmus bzw. eine Computersprache ist noch menschengemacht und damit eine Form von Kommunikation, die interpretationsbedürftig bleibt (vgl. ebd. 44).

9 Es gab selbstverständlich Vorstufen, z. B. die Chronologien und Annalen von Königsgeschlechtern. Auch die Kirchengeschichte des Eusebius oder die Geschichtsbücher der hellenistischen oder biblischen Autoren könnten hier genannt werden. In der Renaissance wurden erstmals Gebäude und Städte gesucht bzw. geschützt, weil man an die antiken Ideale anknüpfen wollte. Die Ausgrabungen Winckelmanns in Pompeij und Herculaneum in der zweiten Hälfte des 18. Jahrhunderts gelten als offizieller Beginn der Archäologie als Disziplin (vgl. Renfrew / Bahn [2005]). 
(1891-1971), der bis heute als Gründervater der Biblischen Archäologie gilt, wichtige Methoden, wie die Tonscherben-Datierung erfand und erste Grabungen vornahm. ${ }^{10}$ Weltruhm erlangte er durch die Authentifizierung der Schriftenfunde von Qumran in den 1940er-Jahren. ${ }^{11}$ Von Seiten der ehemaligen Protektoratsmacht Großbritannien war es die Archäologin Kathleen Kenyon (1903-1978), die, mit ersten Ausgrabungen in Jericho (1950er) und der Altstadt von Jerusalem (1961-1967) sowie innovativen neuen Ansätzen, die zweite prägende Gestalt der Biblischen Archäologie wurde. ${ }^{12}$ Durch die Zuspitzungen im Nahost-Konflikt und die Interessen mehrerer Parteien an der Deutung der Funde sowie durch eine UN-Deklaration ${ }^{13}$, die Grabungen in besetzten Gebieten als „Raubgrabungen“ definiert, sind viele Forschungen unmöglich geworden oder werden ideologisch aufgeladen, sodass die meisten internationalen ForscherInnen in umliegenden Nationen graben oder sich auf erlaubte Orte, z. B. auf kirchlichem Gelände, beschränken. ${ }^{14}$ Dennoch hat Israel selbst mit der Hebrew University, der Tel Aviv University und der Israel Excavation seit

10 Es gab zwar vor Albright ebenfalls Orientforscher und Abenteurer, die biblische Orte suchten und untersuchten, doch sie gingen noch weitgehend unsystematisch oder mit anderen Interessen vor. Zwei wichtige Namen sind in diesem Zusammenhang Edward Robinson (1794-1863) und Alois Musil (1868-1944). Robinson entdeckte bei der Klagemauer Spuren und Überreste des Tempels, weshalb ihm zu Ehren eine seiner Entdeckungn der „Robinson Bogen“ genannt wird. Musil war ein austro-mährischer Theologe, dessen Kartenwerk von Arabia Petraea bis heute als unübertroffen gilt und eine große Anzahl archäologischer Stätten im heutigen Jordanien, in Syrien und im Irak beinhaltet. Zur weiteren Vertiefung sei verwiesen auf Collinet / Hiepel / Veselá / Weigl (2021).

11 Zur Biographie und dem Werk sei verwiesen auf Feinman (2004).

$12 \mathrm{Zu}$ ihrer Rolle und der Entwicklung feministischer Hermeneutik in der Archäologie siehe Davis (2008).

13 UNESCO (1956), Abs. 32.

14 Aus dem deutschen Sprachraum führt vor allem das DEI in Jerusalem unter der Leitung von Dieter Vieweger regelmäßige Grabungen durch, kürzlich erst unter der Erlöserkirche in der Jerusalemer Altstadt und auf dem Gelände der Dormitio, wo Teile der byzantinischen Stadt gefunden wurden. Problematisch sind Grabungen vor allem in Ostjerusalem, am Tempelberg und in jenen Gebieten, die aus 
1948 einige hochproduktive, wenn auch teils umstrittene ArchäologInnen hervorgebracht, z. B. Israel Finkelstein (,Keine Posaunen vor Jericho" [2004]), Benjamin, Amihai und Eilat Mazar (Diese Familie gräbt auf dem Gebiet des heutigen Staates Israel seit 1931.) oder Ehud Netser (Herodes, seine Bauten, sein Grab). ${ }^{15}$

Nach ihrem Selbstverständnis versuchen BibelarchäologInnen die biblischen Orte zu finden, um damit deren Existenz zu belegen, aber auch, um die biblischen Texte kritisch zu überprüfen. Wurden die Mauern von Jericho wirklich niedergerissen? Gab es den Salomonischen Tempel? Wo wurde Jesus gekreuzigt und begraben? Welche Route nahm das Volk Israel beim Exodus? Diese und andere Fragen beschäftigten die Menschen vorrangig bis zum sozialgeschichtlichen Umbruch nach 1968. Nun stellte sich auch die Frage, wie die Menschen zur Zeit Jesu gelebt hatten, was und wie sie kochten oder aßen und welche Stellung Frauen in der Gesellschaft hatten. ${ }^{16}$ Der Blick wechselte von den mächtigen Repräsentationsbauten hin zu den Wohnhäusern und Mistplätzen, zu Wasserkanälen und einfachen Gräbern und Dörfern in der Peripherie; Orte, auf denen auch heute oft noch Wohnhäuser oder sogar religiöse oder politische Gebäude stehen.

Immer wieder stößt man bei dieser Arbeit auf Herausforderungen, bei denen die Digitalisierung ins Spiel kommt. Bereits skizziert ist das Problem der modernen Bebauung oder dass es andere Interessen gibt, die eine Grabung vor Ort unmöglich machen, z. B. Schutz von Kulturgütern, religiöse Gefühle etc. Doch es gibt auch methodische Probleme, von denen zwei im Folgenden vorgestellt werden.

Erstens: Im Wüstensand vergrabene Objekte sind häufig durch das trockene Klima und die Dunkelheit konserviert. In dem Moment, wo sie an die Luft kommen, verderben sie sehr schnell, d. h. Bilder ver-

Sicht des UN-Teilungsplans (1947; 1967) nach dem Sechstagekrieg 1969 und weiteren Konflikten als besetzt gelten.

15 Vgl. für weiterführende Literatur den Überblick von Vieweger (2019), 124-127; $140-142$.

16 Einen guten Überblick bietet Kessler (2006). 
blassen, Schriftrollen zerfallen zu Staub, Metallrollen können nicht mehr entfaltet werden usw. Aus diesem Grund müssen solche Gegenstände schnellstmöglich gesichert und aufgenommen werden. Während dies früher nur mit einer Vermessung per Hand als Zeichnung oder durch eine Fotografie ging, bei der die Belichtung immer auch eine Rolle spielt, kann man heute den gefundenen Gegenstand sofort mit einer Digitalkamera ablichten, die über einen Datumsstempel und ein GPS verfügt, sodass man nicht nur den genauen Standort des Fundes trotz Zeitdruck aufnehmen kann, sondern auch den Sonnenstand im Nachhinein berechnen und so die Belichtung in der Nachbearbeitung anpassen kann. Trotz dieser Vorteile hat das Verfahren noch Schwächen, vor allem bei der Farbechtheit, sodass bei Keramik immer noch das Munsell-Farbschema und die händische Zeichnung wichtig sind. Doch auch hier bietet die Digitalisierung Vorteile. Man braucht nun nicht mehr Lichttische, Zeichenbretter und Millimeterpapier, sondern man kann mit Hilfe eines Tablets und einer App direkt in einen Computer zeichnen. Das Bild ist gespeichert, verzogene Linien können leicht korrigiert werden und sobald die Zeichnung fertig ist, kann der Computer mithilfe einer internen Datenbank die Scherbe auf ein Gefäß hochrechnen und sie nicht nur mit gängigen, sondern mit allen gespeicherten Profilen abgleichen und bestimmen. So ist eine Datierung und Typisierung, die sonst Minuten bis Stunden dauern kann, in Sekundenschnelle erledigt und braucht nur mehr gegengeprüft zu werden. Ein weiterer Vorteil ist die Puzzlefunktion. Da die meisten Gefäße zerbrochen sind, es aber manchmal wichtig ist, ein Gefäß wieder zusammenzukleben, ist der Computer im Vorteil. Er kann alle Funde der Ausgrabung bzw. eines festgelegten Areals miteinander vergleichen und feststellen, welche voraussichtlich zusammenpassen oder zumindest zum gleichen Objekt gehören. ${ }^{17}$

17 Die Quelle dieser Methodik ist ein Kollegen-Gespräch gewesen. Meines Wissens wird diese Software noch entwickelt und erprobt, soll aber bald einsatzbereit sein oder ist es vielleicht schon. 
Zweitens: Um eine Ausgrabung datieren zu können, gibt es in der Biblischen Archäologie drei gängige Methoden. Man bestimmt die Keramikfunde, d. h. man bestimmt, in welcher Zeit und in welcher Gegend der Topf, der Becher oder die Amphore hergestellt bzw. benutzt wurde. Zweitens kann man mit der $\mathrm{C}^{14}$-Methode Textilien, Hölzer und Ähnliches untersuchen, was aber nur sehr grobe Datierungen erlaubt. ${ }^{18}$

Drittens werden bei Grabungen die archäologischen Schichten von oben nach unten und damit von der chronologisch jüngsten zur ältesten abgetragen. Dadurch werden sie in ein zeitliches Verhältnis zueinander gesetzt, das man Stratigrafie nennt. Das Problem bei diesem Grabungsvorgang ist, dass man damit zwar weiß, auf welcher Ebene ein Fund gemacht wurde, d. h. welche Siedlung auf welcher errichtet wurde, und sie so datieren kann. Aber die weiter oben liegenden Schichten müssen dafür abgetragen und somit zerstört werden. Wenn die gewünschte Schicht gefunden wird, z. B. die Zeit des babylonischen Exils (6. Jh. v. Chr.) oder eine angenommene Blütezeit des Ortes, dann wird diese freigelegt und die Grabungen hören oft auf. Damit sind die jüngeren Schichten zerstört und die älteren, darunterliegenden, bleiben unerforscht. ${ }^{19}$ Abhilfe schaffen hier neuerdings die aufsehenerregenden Methoden der Laserarchäologie. Unter den weltweiten Vorreitern ist das Ludwig Boltzmann Institut Archaeological Prospection and Virtual Archaeology in Niederösterreich unter der Leitung von Prof. Wolfgang Neubauer. ${ }^{20}$ Mithilfe eines speziellen Ultraschall- und Lasersystems wird der Boden mit einer Art Echolot beschallt und anschließend die Störfrequenz, also Sandkörner oder

$18 \mathrm{Zu}$ den gängigen Datierungsmethoden vgl. Frevel (2016), 37-40; Vieweger (2019), 139.

19 Vgl. Vieweger (2019).

20 https://archpro.lbg.ac.at/ - Bekannt sind etwa die Vermessung von Stonehenge (2010; 2014; 2020), die Entdeckung eines Schiffsfriedhofs im Eis (2010; 2015; 2020), die Gladiatorenschule von Carnuntum (2011) oder die Funde unter der Spanischen Hofreitschule im aktuellen Jubiläumsjahr. Die Gladiatorenschule kann man sich anschauen in Klein (2019), 94-97. 
Erdklumpen und Steine, aus dem Bild herausgerechnet. Zurück bleibt ein klares Bild von den Strukturen in mehreren Metern Tiefe, das von einer Münze bis zu einem Gebäude alles anzeigen kann. Nach dieser ersten Auswertung kann dann das Team oder der Financier entscheiden, ob und vor allem exakt wo und was ausgegraben werden soll, oder ob es genügt zu wissen, wo das Objekt liegt. Dies bedeutet, dass verderbliche Funde nicht mehr zwingend geborgen werden müssen. Es ist nun also möglich, alle Schichten einer Ausgrabung voneinander zu unterscheiden und - ohne eine andere Schicht zu zerstören zu erfahren, wie weit zurück die Besiedlung eines Ortes geht, wann seine Blütezeit war und wo die lohnendsten Funde sind. Auf diese Weise könnte man etwa auch am Tempelberg in Jerusalem oder unter der Grabeskirche archäologische Untersuchungen vornehmen, ohne Welterbe zu zerstören oder religiöse Gefühle (übermäßig) zu verletzen. Ein weiterer Vorteil ist die Möglichkeit, aus den erhobenen Daten ein 3D-Modell anzufertigen. ${ }^{21}$ Einerseits können Scherben oder ganze Gefäße in einen 3D-Scanner gelegt und so ohne Umweg dreidimensional gespeichert werden. Andererseits sind Lasergeräte und Drohnen in der Lage, ganze Gebäude oder sogar Städte zu vermessen und davon ein maßstabsgetreues Modell zu erzeugen. ${ }^{22}$ Seit Neustem ist es sogar möglich, das entstandene Modell mit einem 3D-Drucker auszudrucken, sodass man innerhalb weniger Tage ein vollständiges und maßstabsgetreues Modell einer Stadt oder ein hochdetailliertes Gebäude erzeugen kann. ${ }^{23}$

\section{Alles auf einen Klick. Bibelkunde digital}

Um Exegese treiben, aber vor allem um sie unterrichten zu können, braucht es eine Vielzahl von Hilfsmitteln: Handschrifteneditionen zur Textbestimmung, Wörterbücher und Grammatiken für die Sprachanalyse, Konkordanzen, Lexika und Kommentare für die Inhaltsana-

\footnotetext{
21 Vgl. Kai-Browne (2019), 62-65.

22 Vgl. Höhler-Brockmann (2019), 24-27.

23 Vgl. Gerlach / Lindstaedt / Schnelle (2019), 35.
} 
lyse, Atlanten, Reiseführer und andere Karten- und Bildwerke um einen visuellen Eindruck vom biblischen Israel und seiner Zeit zu haben. ${ }^{24}$ Analog bedeutet dies, dass es riesige Bibliotheken braucht, große Büros, zahlreiche HilfsarbeiterInnen sowie kosten- und zeitintensive Neuanschaffungen. Digital lässt sich eine Vielzahl dieser Probleme lösen. Eine Bibliothek passt heute auf einen USB-Stick, die Konkordanz-Beziehungen zwischen Texten kann man mit einer Bibelsoftware bestimmen und sogar statistisch darstellen lassen. Karten können in Sekundenschnelle gewechselt und innerhalb kürzester Zeit bearbeitet und in verschiedenen Versionen gespeichert werden. Gibt es Neuerungen an Editionen oder Kartenwerken, so lassen sie sich per Update unmittelbar nach Entdeckung bzw. Editierung herunterladen und man muss nicht warten, bis ein neuer Ergänzungsband erscheint oder ausreichend Material für eine Neuauflage vorhanden ist. Es gibt mittlerweile sogar die Software Transkribus, die an der Universität Innsbruck mitentwickelt wurde, welche in der Lage ist, Handschriften zu lesen und in Unicode umzuwandeln. ${ }^{25}$ Selbst die Kostenfrage hat sich gewandelt, da Software und E-Books zwar nicht ganz billig sind, in der Regel jedoch wesentlich günstiger als die Anschaffungskosten wissenschaftlicher Literatur. ${ }^{26}$ Die Digitalisierung hat in diesem Bereich zu einer enormen Effizienzsteigerung geführt, die noch

24 Aus Platzgründen kann hier nicht die gesamte Geschichte der Exegese von der Selbstauslegung und dem Midrasch über Origenes bis heute vorgestellt werden. Wichtige Stationen waren etwa die Mnemotechniken Hugo von St. Victors im Hochmittelalter und der Umstieg auf die Schriftstellenverzeichnisse, wie sie Illich (2014), 33-54, beschreibt, später dann die Ausbildung der Nationalsprachen und mit ihnen verbunden die Entstehung von Grammatiken, die historische Sprachforschung und Kartographie u. v. m.

25 Vgl. Clivaz (2017), 51; Clivaz selbst arbeitet derzeit an einem Projekt mit, welches die wichtigsten neutestamentlichen Handschriften, die in der sogenannten Nestle-Aland Ausgabe stehen, digital verfügbar und vergleichbar macht, um sie dann mithilfe von Transkribus im textkritischen Apparat zu hinterlegen. Die Homepage findet sich unter https://transkribus.eu/lite/de.

26 Vgl. zu den Problemen zwischen freeware und high-end Bibelsoftware die Ausführungen bei Hiepel / Niggemeier (2021), 206. 
einen weiteren Vorteil hat: Publikationen und Bilder lassen sich per E-Mail in Echtzeit verschicken, ohne dass Transportgebühren oder Lieferzeiten anfallen; online-Publikationen sind sogar dauerhaft und teilweise gratis weltweit abrufbar, sofern es einen Internetanschluss gibt, sodass es möglich wird, globale Studien einzusehen und auch zu verteilen, ein Vorteil besonders für ärmere Regionen der Erde, sofern man darauf achtet, keine neo-koloniale Bildungsschwemme zu produzieren. Derzeit wird außerdem an Übersetzungssoftwares gearbeitet, die eine neue Form von lingua franca ermöglichen: das Binär oder auch Basic. Ähnlich dem Babel-Fisch aus dem Roman „Per Anhalter durch die Galaxis“ (Douglas Adams, 1979) reicht es, in ein digitales Endgerät zu sprechen und das Gegenüber erhält eine Übersetzung in Wort oder Schrift von diesem Text in einer Sprache, die sie / er selbst versteht.

Die Schwäche, die diesem ganzen System anhängt, ist die Fähigkeit zur Interpretation und Definition. Digitale Netzwerke und Komplexe arbeiten mit ihrer eigenen Sprache und entscheiden nach Algorithmen. Diese sind zwar lernfähig nach einem bestimmten vorgegebenen Modus, der etwa für personalisierte Werbung oder Informationsblasen auf Social Media verantwortlich ist. Dennoch ist ihr Ziel vor allem Komplexitätsreduktion und eine Entscheidung basierend auf statistischer Wahrscheinlichkeit. Die Digitalisierung produziert insofern ein unzuverlässiges Medium, weil es gerade nicht in der Lage ist, seine binäre Dualität aufzulösen, d. h. zwischen weiß und schwarz Grauschattierungen aufrecht zu erhalten, z. B. die Polysemie von Wörtern mitzudenken oder seltenen Formen Vorrang zu geben, wenn sie nicht wiederum als eigenes Muster erkannt und programmiert worden sind. ${ }^{27}$ Ein weiterer Aspekt ist die biographische Komponente. Der Algorithmus basiert auf den Annahmen, die ein Team von InformatikerInnen vorgegeben hat. Diese befassen sich in der Regel nicht mit einem langwierigen Theologiestudium, da sie

27 Auf diesen Tunnelblick verweisen auch Harrich / Hiepel (2021), 95; Campbell (2013). 
andere Expertisen ausgeprägt haben und können damit nicht verhindern, dass ihr Algorithmus nur begrenzt in der Lage ist, geisteswissenschaftlich zu denken, geschweige denn zu theologisieren oder gar eine spirituelle Textdeutung anzubieten.

Aus diesem Zwiespalt, dem auf der anderen Seite die häufig mangelnde Kompetenz von GeisteswissenschaftlerInnen im Umgang mit der Digitalisierung gegenübersteht, wächst seit einigen Jahrzehnten ein neues Paradigma, das man „Digital Humanities“ (digitale Geisteswissenschaften) nennt. ${ }^{28}$ Ein Vorreiter dieser Bewegung war der in Bozen geborene Jesuit Roberto Busa (1913-2011). Als Thomasforscher plante er als Langzeitprojekt den Index Thomisticus, eine Konkordanz zu Thomas von Aquin, die 56 Bände und 70.000 Schlagwörter umfassen sollte. Die geschätzte Arbeitszeit betrug, wenn per Hand ausgeführt, 40 Jahre. Busa beschäftigte sich daraufhin mit den Möglichkeiten, die neuere Computersoftware anbot, und lernte programmieren. Nachdem er das System mit Daten gefüttert hatte, gelang es ihm, sein Gesamtprojekt in sieben Jahren abzuschließen, d. h. in etwas mehr als einem Sechstel der veranschlagten Zeit. ${ }^{29}$ Mittlerweile gibt es, inspiriert von Busas Arbeit, unzählige Digitalisierungsprojekte auf der ganzen Welt, die von staatlicher und universitärer Seite stark gefördert werden. Man findet nun Kongressbibliotheken, Zeitdokumente wie Fotos, Radioansprachen oder Videos im Internet, aber auch Bücher, deren Urheberrecht erloschen ist, Datenbanken und Indices, wie die für die Theologie wichtigen IxTheo oder BILDI/ KALDI, welche unter anderem von der Theologischen Fakultät Innsbruck betreut werden. Darüber hinaus entwickeln sich Projekte zur Langzeitarchivierung oder die Erstellung von Lexika, die auf dem Wiki-System basieren (am berühmtesten ist die Enzyklopädie Wikipedia), ständig weiter und wachsen an. ${ }^{30}$

\footnotetext{
28 Vgl. Hiepel / Niggemeier (2021), 201. Zur hermeneutischen Komponente dieses Paradigmas in Bezug auf Religion vgl. den Sammelband von Campbell / Altenhofe (2015).

29 Vgl. Clivaz (2017), 45; Jones (2016).

30 Vgl. Garcés / Heilmann (2017), 29-52.
} 
Die interdisziplinäre Kooperation von Informatik, Webdesign und Geisteswissenschaften ist nicht nur in der Forschung wichtig, sondern sie erlaubt darüber hinaus den Lehrenden, wieder näher an die Lebenswelt der Studierenden zu rücken. War in früheren Zeiten nicht nur der Erfahrungs- sondern auch der Wissensvorsprung älterer Menschen zumindest im Klassenraum unhinterfragt, können SchülerInnen heute einfach zum Handy greifen und die Aussagen der Unterrichtenden überprüfen bzw. aktuellere Informationen, als sie in der Morgenzeitung standen, abrufen. Jene jungen Menschen, die nach der Einführung des flächendeckenden Breitbandanschlusses um die Jahrtausendwende geboren wurden, bezeichnet man als „Digital Natives", d. h. sie bewegen sich im digitalen Raum mit der gleichen oder sogar noch größeren Selbstverständlichkeit, mit der sie auch in der physischen Welt unterwegs sind. Für sie gibt es ganz eigene Herausforderungen und einen Erfahrungsvorsprung, der zunächst einmal eingeholt werden muss. ${ }^{31}$

Um dieser Gruppe in der bibelwissenschaftlichen Lehre gerecht zu werden, schlagen Hiepel und Niggemeier von der WWU Münster ein exploratives Lehrmodell vor. ${ }^{32}$ Studierende müssen nun nicht mehr erst nach Israel fliegen, um die Landschaft kennenzulernen. Sie können stattdessen mit dem Online-Format Google Earth von zu Hause aus per Satellitenbild und Fotoaufnahmen (street view) das Land anschauen oder durch die Straßen Jerusalems spazieren und dazu Übungen absolvieren. Derzeit wird außerdem daran geforscht, virtuelle Realitäten so zu programmieren, dass man in ihnen nicht nur in den archäologischen Stätten steht, sondern per 3D-Modell auch durch sie hindurchwandern kann, als wäre man im Jahr 600 v. Chr.

Ein solches Modell hat viele Vorteile, gerade in Zeiten wie der Covid-19 Pandemie, in der Reisen nahezu unmöglich ist. Doch es gibt auch einen entscheidenden Nachteil, der aller Digitalität anhaftet: den Verlust der Leiblichkeit. Es ist von Vorteil, wenn Studierende

\footnotetext{
31 Vgl. Theis (2019), 2-10; Hiepel / Niggemeier (2021), 204.

32 Vgl. Hiepel / Niggemeier (2021), 211.
} 
ohne Zeit- und Geldaufwand und ohne den ökologischen Schaden eines Fluges Israel besuchen können. Sie spüren dabei jedoch weder die Aufregung der Fernreise, noch erleben sie die Entfernung und Überwindung mehrere tausend Kilometer zeitlich und räumlich. Es fehlt ihnen gänzlich der Kulturschock, wie es ist, in ein jüdisch-muslimisches Land zu kommen, in dem der Muezzin und nicht die Kirchenglocken zum Gebet rufen. Sie riechen nicht die Kombination aus Gewürzen, Gemüse, Schweiß und in der Sonne hängendem Fleisch, welche die Märkte ausmacht, und spüren auch nicht die Hitze auf der Haut. Sie hören nicht die Fremdheit der Sprachen, sehen nicht die militärischen Auswirkungen des gespaltenen Landes und erleben nicht in gleicher Weise die Präsenz der heiligen Stätten, den Glauben der Menschen, die Gnadenlosigkeit der Wüste und die Freude, Wasser zu sehen.

\section{Logout: analoges Fazit}

Dieser kurze Beitrag setzte sich zum Ziel, die Vor- und Nachteile der Digitalisierung für die bibelwissenschaftliche Arbeit anhand einiger Beispiele in den Blick zu nehmen. Methodisch steht außer Frage, dass die Digitalisierung einen völlig neuen und verbesserten Werkzeugkasten für die Analyse und Darstellung von Daten gibt. Die fortschreitende Bewegung der Digital Humanities erlaubt auf Dauer, sofern sie nicht kapitalistischen oder kolonialen Vorhaben anheimfällt, mehr Zugang zu Bildungsressourcen und ermöglicht einen globalen Austausch in Echtzeit. Viele Routine-Arbeiten können schneller, billiger und weniger raumintensiv erledigt werden, sodass mehr Zeit für die Auswertung und Diskussion bleibt.

Der Nachteil der Digitalisierung ist die permanente Übersetzung der physischen Welt in eine binarisierte Computerform, die von Servern, hohem Energieaufkommen und vielen anderen Faktoren abhängig ist. Jede Übersetzung führt zu einer Bedeutungsverschiebung, die schon im Übergang von der Mündlichkeit zur Schriftlichkeit gesehen werden kann. Der Erfahrungsbonus, den jüngere Generationen mit 
digitalen Endgeräten haben, gleicht nicht aus, dass sie einen verantwortungsvollen Umgang mit diesen Geräten lernen müssen und erfahrenere Menschen brauchen, die ihnen helfen, zuverlässige Quellen von unbrauchbaren zu unterscheiden und sich im mass data zurechtzufinden.

Die Bibelwissenschaft ist es gewöhnt, sich der hermeneutischen und methodischen Möglichkeiten ihrer Zeit zu bedienen und sie wird es auch weiterhin tun. Dabei ist es jedoch von bleibender Bedeutung, dass sie sich ihrer Tradition und Genese bewusst bleibt, um ihrem eigentlichen Auftrag gerecht zu werden: das Wort Gottes verstehen, verstehbar zu halten und zu verkünden.

\section{Literatur}

$b p b$ (2021), Digitalisierung (Informationen zur politischen Bildung 344). Bonn.

Campbell, Heidi A. (2013) (Hg.), Digital Religion. Understanding Religious Practice in New Media Worlds. London.

Campbell, Heidi A. / Altenhofen, Brian (2015), Methodological Challenges, Innovations and Growing Pains in Digital Religion Research. In: Sariya Cheruvallil-Contractor / Suha Shakkour (Hg.): Digital Methdologies in the Sociology of Religion. London, 1-12.

Clivaz, Claire (2017), Die Bibel im digitalen Zeitalter. Multimodale Schriften in Gemeinschaften. In: ZNT 20, 35-57.

Collinet, Bendikt J. / Hiepel, Ludger / Veselá, Martina / Weigl, Michael (2021) (Hg.), Alois Musil. Perspektiven auf eine vielschichtige Persönlichkeit (InOr 7). Münster.

Davis, Miriam (2008), Dame Kathleen Kenyon: Digging Up the Holy Land. Walnut Creek, CA.

Feinman, Peter D. (2004), William Foxwell Albright and the Origins of Biblical Archaeology. Berrien Springs, MN.

Frevel, Christian (2016), Geschichte Israels. Stuttgart.

Garcés, Juan / Heilmann, Jan (2917), Digital Humanities und Exegese. Erträge, Potentiale, Grenzen und hochschuldidaktische Perspektiven. In: Forum Exegese und Hochschuldidaktik. Verstehen von Anfang an 2/2, 29-52. 
Gerlach, Iris / Lindstaedt, Maren / Schnelle, Mike (2019), Der Grat Be'al Gebri. Ein palastartiger Monumentalbau aus dem frühen 1. Jt. v. Chr. im Hochland Äthiopiens. In: WBG (Hg.), Auferstehung der Antike. Archäologische Stätten digital rekonstruiert. Darmstadt, 32-35.

Harrich, Simon / Hiepel, Ludger (2021), Computational Thinking. Ein Ansatz für die Theologie? In: Ludger Hiepel / Andre Burke u. a. (Hg.), Theologiestudium im Digitalen Zeitalter. Stuttgart, 87-96.

Hasse, E. Sebastian (2013), Von der Offenbarung ins Web 2.0: die Bibel im digitalen Zeitalter - Impulse für eine Medientheologie. Gießen.

Hiepel, Ludger / Niggermeier, Volker, Nicht auf Sand gebaut. Bibelwissenschaftliche Lehre im digitalen Zeitalter. In: Ludger Hiepel / Andre Burke u. a. (Hg.), Theologiestudium im Digitalen Zeitalter. Stuttgart, 201-214. Höhler-Brockmann, Hajo (2019), Erfassung von 3D-Daten im archäologischen und kulturhistorischen Kontext. In: WBG (Hg.), Auferstehung der Antike. Archäologische Stätten digital rekonstruiert. Darmstadt, 24-27.

Illich, Ivan (2014), Im Weinberg des Textes. Als das Schriftbild der Moderne entstand. 2. Auflage. München.

Jones, Steven E. (2016), The priest and the punched cards. Roberto Busa S. J. and the emergence of Humanities Computing. London.

Kai-Browne, Arie (2019), Visualisierung von 3D-Modellen. In: WBG (Hg.), Auferstehung der Antike. Archäologische Stätten digital rekonstruiert. Darmstadt, 62-65.

Kessler, Rainer (2006), Sozialgeschichte des Alten Israel. Eine Einführung. Darmstadt.

Klein, Michael u. a. (2019), Virtuelle Rekonstruktionen in Carnuntum. Neue Entdeckungen zur Stadtgeschichte der römischen Donaumetropole. In: WBG (Hg.), Auferstehung der Antike. Archäologische Stätten digital rekonstruiert. Darmstadt, 94-97.

Renfrew, Colin / Bahn, Paul G. (2005), Archaeology - Theories, Methods and Practice. 5. Auflage. London.

Schweiger, Wolfgang (2017), Der (des)informierte Bürger im Netz. Wie soziale Medien die Meinungsbildung verändern. Wiesbaden.

Theis, Joachim (2019), Digital Natives und die Bibel - eine unmögliche Verbindung? In: BiKi 74, 2-10.

UNESCO (1959), Recommendation on International Principles Applicable to Archaeological Excavations (5. 12. 1956). http://portal.unesco.org/ 
en/ev.php-URL_ID $=13062 \& U R L \_D O=D O \_T O P I C \& U R L \_S E C-$ TION=201.html [7. 5. 2021].

Vieweger, Dieter (2019), Biblische Archäologie. In: Dieter Vieweger / Siegfried Kreuzer u. a. (Hg.), Proseminar Altes Testament. Ein Arbeitsbuch. 3. Auflage. Stuttgart, 124-146. 